\title{
Visual Snow in Two Patients after Hospitalization for Bacterial Illness
}

\author{
Mohammad Z Siddiqui ${ }^{1}$ and Joseph Chacko ${ }^{2 *}$ \\ ${ }^{1,2}$ Department of Ophthalmology, Jones Eye Institute, University of Arkansas for Medical Sciences, Little Rock, USA
}

*Corresponding author: Joseph Chacko, Department of Ophthalmology, Jones Eye Institute, University of Arkansas for Medical Sciences, Little Rock, Arkansas 72205, USA
Received Date: October 11, 2020

Published Date: December 02, 2020

\begin{abstract}
Visual snow is a phenomenon with an unclear etiology that was first described over twenty years ago in patients with migraine. The main symptoms patients experience includes a constant positive visual disturbance of flickering dots, resembling snow. We present two patients that developed visual snow after being hospitalized for bacterial illnesses. Patient 1 is a 28 -year-old Caucasian female who was 29 weeks pregnant at presentation for a complaint of visual snow in both eyes that she developed shortly after a previous miscarriage due to bacterial sepsis. Patient 2 is a 27-year-old male who reported seeing constant static spots in both eyes for the past year that developed after being hospitalized for two weeks due to bacterial pneumonia and sepsis. There is a clear temporal relationship between bacterial illnesses and the development of visual disturbances in the presented two patients. Further research needs to be conducted pertaining to the neurological and neuro-psychiatric association of visual snow and its association with bacterial sepsis.
\end{abstract}

Key Words: Visual Snow; Sepsis; photopsia; Migraine

\section{Introduction}

Visual snow is a phenomenon with an unclear etiology that was first described over twenty years ago in patients with migraine [1]. Clinical criterion for this condition proposed by Schankin et al includes seeing dynamic and continuous static in the entire visual field for greater than 3 months [2,3]. Additionally, palinopsia, enhanced entoptic phenomena, photophobia, or nyctalopia may be present [2,3]. Visual snow can be associated with stress, depression, and illicit drug use; however, no clear causative source has been identified $[3,4]$.

The main defining symptom that patients describe is the constant positive visual disturbance of flickering dots, resembling snow. The symptoms can persist for years. A large systematic review of cases done by Puledda et al found that $25 \%$ of patients had symptoms for as long as they could remember from childhood and $75 \%$ had symptom onset around the second decade of life [5]. There is usually either a family or personal history of migraines. Also, asso ciated anxiety can be found in $44 \%$ of patients [5]. Visual snow was thought be part of the spectrum of migraine aura in the past; however, increasing medical evidence suggests that visual snow is likely a unique entity [6]. It has been noted that visual snow patients with a history of migraines experience more severe symptoms such as photophobia, than those without a history of migraines [2].

We present two patients that had severe bacterial illness and hospitalizations with subsequent development of visual snow.

\section{Case Presentation}

Patient 1 is a28-year-oldCaucasian female who was 29 weeks pregnant at presentation to our clinic with the complaint of visual snow in both eyes. The patient described her visual symptoms as constant flickering in both eyes with associated floaters and photophobia for one year. She had a past medical history of migraines since age 7 , gestational diabetes, neurocardiogenic 
syncope, and a small macular scar from childhood in her left eye. There was a family history of migraines in her mother. The visual snow symptoms began after she was hospitalized in the United Kingdom during a previous pregnancy for a critically high fever for four days and complications from gram-negative bacteremia. At the time of hospitalization, she was 12 weeks pregnant and subsequently had a miscarriage at 18 weeks. Her eye exam showed a corrected visual acuity of 20/20 OU. Intraocular pressures were 17 OU. Slit lamp exam showed trace posterior sub capsular cataract and a tiny fovea defect in the left eye. Color vision was normal. 2-year follow-up revealed no change in symptoms.

Patient 2 is a27-year-oldCaucasian male who complained of seeing constant static spots in both eyes for one year. He described the spots as small and white, resembling snow. He denied headaches and diplopia. He had a family history of migraines. The visual disturbances started while he was hospitalized for two weeks in Russia for pneumonia and sepsis while studying abroad one year prior. During this hospitalization, he lost $35 \mathrm{lbs}$. due to the pneumonia and sepsis. The patient described the static spots as constant since his prolonged hospitalization. His exam showed an uncorrected visual acuity of 20/30 OU. Intraocular pressures were normal. Slit lamp and funduscopic exam did not show any abnormalities. The patient was prescribed $10 \mathrm{mg}$ amitriptyline once per day and advised to return in 6 months. At the 6-month followup visit, the patient reported that the white spots were still present, but not as noticeable. He did not take the amitriptyline because of potential side effects. His exam was normal.

\section{Discussion}

As more cases are reported, evidence is being formulated suggesting that visual snow is a complex neurological syndrome. The typical description of symptoms in patients with visual snow and persistence even during times with eyes closed is suggestive that this syndrome is a biological phenomenon [5].

The pathophysiology of visual snow is not distinctly defined. There are a number of proposed hypotheses in the recent literature. Thalamo-cortical dysrhythmia due to dysfunctional neuronal excitability and an impaired habituation response is one of the leading theories for visual snow [4]. Interestingly, some patients have reported improvement of their symptoms with yellow-blue color filters. This supports the concept that the dysfunction is neurological and possibly related to color with involvement of the magnocellular processing pathway [4].

Brain hyper-metabolism in the supplementary visual cortex of Brodmann area 19 (bilateral lingual gyrus) has been described in patients with visual snow through functional brain imaging with [18F] FDG positron emission tomography [2,6]. Rastogi et al theorize this to be a problem of higher visual processing.
To our knowledge, there have not been previous cases reported in the literature of severe bacterial illness and hospitalization leading to visual snow. It is clear that the onset of visual symptoms our two patients experienced began after significant illnesses with high fevers and prolonged hospital courses. Although this association may be coincidental, we believe that the stress the body experienced contributed to the onset of the visual snow. Sepsis has been known to cause brain dysfunction, and those who survive severe sepsis can often develop long term cognitive impairments and behavioral problems [7]. Sonneville et al have described MRI studies that have associated septic shock with new and acute cerebrovascular lesions and white matter abnormalities [7].

Visual snow is likely both a neurological and neuropsychiatric process. Both our patients had severe physical and physiologic stressors, with patient 1 having a miscarriage associated with systemic infection, and patient 2 having lost 35lbs. due to severe pneumonia and sepsis. Patient 2 subsequently suffered anxiety due to his prolonged illness in a foreign country away from home. Further research needs to be conducted to characterize the association of severe bacterial illness and stressors with the development of visual snow.

\section{Declaration of Interest Statement}

The authors report no conflict of interest.

\section{Data Availability Statement}

No data was used to support this study.

\section{Funding Statement}

Authors did not receive funding for this study.

\section{Acknowledgement}

Jones Eye Institute, University of Arkansas for Medical Sciences.

\section{References}

1. Liu GT, Schatz NJ, Galetta SL, Volpe NJ, Skobieranda F, et al. (1995) Persistent positive visual phenomena in migraine. Neurology 45(4): 664-668.

2. Schankin CJ, Viana M, Goadsby PJ (2017) Persistent and Repetitive Visual Disturbances in Migraine: A Review. Headache 57(1): 1-16.

3. Schankin C, Maniyar F, Digre K, Goadsby P (2014) 'Visual snow' - a disorder distinct from persistent migraine aura. Brain 137(Pt 5): 1419 1428.

4. Lauschke JL, Plant GT, Fraser CL (2016) Visual snow: A thalamocortical dysrhythmia of the visual pathway? J Clin Neurosci 28: 123-127.

5. Puledda F, Schankin C, Digre K, Goadsby PJ (2018) Visual snow syndrome: what we know so far. Curr Opin Neurol 31(1): 52-58.

6. Rastogi RG, Vander Pluym J, Lewis KS (2016) Migrainous Aura, Visual Snow, and "Alice in Wonderland" Syndrome in Childhood. Semin Pediatr Neurol 23(1): 14-17.

7. Sonneville R, Verdonk F, Rauturier C, Fabrice Chretien, Tarek Sharshar, et al. (2013) Understanding brain dysfunction in sepsis. Ann Intensive Care 3(1): 15 